\title{
LA TABLETA DE CERRO MACARENO: ANÁLISIS EXPLORATORIO DE DATOS EN TORNO A UNA PIEZA DE ARQUEOLOGÍA
}

\author{
THE CERRO MACARENO TABLET: EXPLORATORY DATA \\ ANALYSIS OF AN ARCHAEOLOGICAL FIND
}

\author{
ÍÑIGO SÁEZ URIBARRI*
}

\begin{abstract}
Resumen: La Tableta de Cerro Macareno es una pieza de barro cocido con numerosas muescas grabadas que presenta severas incógnitas cronológicas y funcionales. Se ha estudiado la información manifestada a través de las muescas con el objeto de aclarar su función. Tras un proceso de codificación se han utilizado varias técnicas estadísticas. Se ha demostrado que las muescas no se distribuyen al azar y pierde peso la idea de que represente algún tipo de escritura. Adquiere fuerza la consideración de que la pieza constituya un registro de algún suceso distribuido en el espacio y/o en el tiempo.
\end{abstract}

Palabras clave: Análisis multivariable, análisis exploratorio, tableta cuneiforme.

La Tableta de Cerro Macareno es una pieza de barro cocido expuesta en el Museo Arqueológico de Sevilla. Contiene grabada una forma rectangular dividida por líneas horizontales y verticales, en 8 filas y 14 columnas. Esto da lugar a 112 casillas que contienen muescas horizontales, verticales o espacios en blanco; es decir, aparecen representadas en estas casillas al menos tres tipos de elementos diferentes.

En la Figura 1 se recoge un esquema realizado por Fernández, F. y col. (1979: 59) sobre el que se ha basado este estudio.

Fernández, F. y col. (1979: 59) describen la localización geográfica del yacimiento en que fue encontrado,

\footnotetext{
* Fundación Vasca para la Formación Profesional Continua-Hobetuz.
}

\begin{abstract}
Cerro Macareno's tablet is a piece of fired clay with many incisions that pose puzzling chronological and functional queries. The information provided by the incisions has been studied with the purpose of elucidating their function. Following a coding process, several statistical techniques have been used. It has been proven that incisions are not randomly distributed. And while the idea that they might stand for some kind of writing loses weight, the fact that the piece may be a record of some event taking place over the time and/or space horizon is gaining importance.
\end{abstract}

Key words: Multivariable analysis, Exploratory analysis, cuneiform tablet.

el contexto en el que se produjo el hallazgo, así como los condicionantes en los que éste se produjo.

En relación a esta última cuestión, resulta difícil situarla cronológicamente debido a que fue hallada por un obrero que dice haberla recogido a siete metros de profundidad y en tierras que habían sido remozadas con máquinas (Fernández, F. y col. 1979: 59). Pero éste no es el único interrogante que plantea, dadas las peculiaridades de su diseño.

El yacimiento del Cerro Macareno en el que fue encontrada la Tableta, se haya ubicado en el término municipal de La Rinconada, a pocos kilómetros de Sevilla. Se asienta en plena llanura aluvial del Guadalquivir. El yacimiento es una formación que tiene cerca de 10 metros de altura sobre el nivel de las tierras circundantes. Está originado por la 


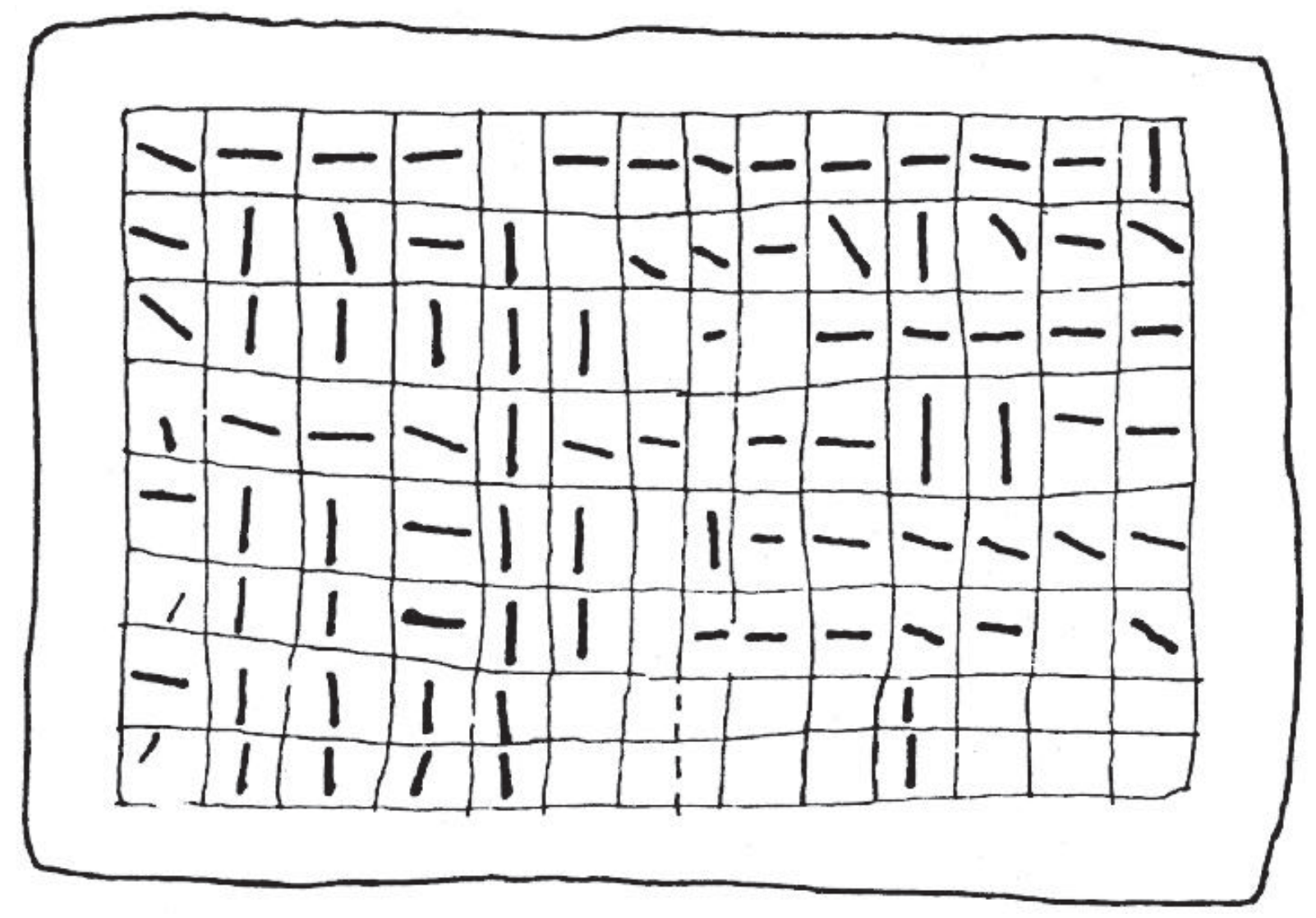

Fig. 1. Esquema de la Tableta de Cerro Macareno al doble de su tamaño original

acumulación de restos arquelógicos y es similar a otros que se encuentran a todo lo largo del río Guadalquivir.

En la base del cerro aparece una capa de arcilla roja, de unos $50 \mathrm{~cm}$. de potencia. En contacto con la parte inferior de esta capa aparece otra más débil de unos 20 $\mathrm{cm}$. de altura. Por encima del nivel de las arcillas se acumulan los restos arqueológicos. Éstos se extienden, por lo que conocen los autores, desde una etapa avanzada del bronce final hasta la época romana.

En cuanto al contexto en que se produce el hallazgo, es necesario recordar que el descubrimiento del Cerro como yacimiento coincidió prácticamente con el principio de su sistemática destrucción para poder explotar sus gravas depositadas por el río Guadalquivir. La destrucción del yacimiento duró meses hasta el inicio de un trámite de expropiación forzosa. Un acuerdo con los propietarios permitió la donación de la parte del yacimiento en la que todavía no había intervenido maquinaria.

En cuanto a su aspecto, la Tableta no da la impresión de ser un objeto ornamental en sentido estricto, por dos razones fundamentalmente:
- Las líneas representadas en las casillas no se distribuyen con una estética geométrica evidente.

- No se representan aparentemente formas ornamentales reconocibles.

Otras piezas arqueológicas presentan alguna de estas características y quizá compartan una relación funcional con ella. Este el es caso de las cucharas o paletas de barro cocido de tradición eneolítica del poblado bajo del Carambolo (Carriazo 1973: 215).

Un plato hallado en la necrópolis de la Edad de Bronce de Los Algarbes contiene un dibujo similar con forma de rejilla, con una serie de rectas paralelas que se cortan perpendicularmente. Este esquema es frecuente en la cultura a la que pertenece esta necrópolis según Posac C. (1975: 118).

También cabe citar, por el empleo de signos sencillos, las tablillas sumerias arcaicas halladas en Uruk. Son pequeñas placas de arcilla cocida de forma generalmente rectangular y con sus dos caras principales alabeadas. 
Los signos en ellas grabados aparecen inscritos sobre una cuadrícula, mediante presión con un determinado instrumento. A los lados de estas marcas se encuentra también uno o varios dibujos más o menos esquemáticos hechos con buril, representando seres u objetos de todo tipo. Según G. Ifrah (1998: 203), las marcas grabadas corresponden a las diferentes clases de unidades de la numeración escrita sumeria en su grafía arcaica.

En el caso de la Tableta, son varias las hipótesis formuladas en torno a esta pieza:

- Según C. Fernández-Chicarro (1974: 31) podría tratarse de un tipo de escritura relacionada con el cuneiforme y que exista la posibilidad de que se trate de una Tableta de archivo.

- El Doctor Gjerstadt (Fernández, F. y col. 1979: 58-59) cree probable que sea algo romano.

- Para Fernández, F. y col. (1979: 59) también puede deberse al capricho del alfarero.

- Asimismo, Fernández, F. y col. (1979: 60) apunta la posibilidad de que sea un inventario o algo similar.

Si la función acertada fuera de registro de algún tipo de objeto o de algún tipo de sucesos, cabe esperar que la información contenida en las casillas guarde entre sí algún tipo de interdependencia.

En el caso de que fuera el capricho de un alfarero, podríamos pensar que el contenido de las casillas no sigue ningún criterio, es decir, que su distribución sea, en el peor de los casos, aleatoria. De cualquier forma es necesario plantear como hipótesis de trabajo la siguiente pregunta: ¿Los objetos que aparecen representados en la Tableta están puestos ahí al azar?

Una distribución al azar haría poco interesante a este objeto, al menos desde el punto de vista del estudio de la información en él contenido. En otro caso la realización de los correspondientes análisis de datos con fines puramente exploratorios pueden acercarnos a una mejor comprensión de la función de la Tableta.

Para alcanzar este objetivo, los análisis multivariantes se nos ofrecen como potentes armas exploratorias de patrones y tendencias. Así opinan Cereijo M. A. y col. (1991: 144). De acuerdo con estos autores, estos análisis muestran interpretaciones que deben ser tratadas como modelos provisionales. Ahondando en este argumento, J. A. Barceló (1991: 42) entiende por interpretación el procedimiento que permite asociar a unas unidades de información empíricas concretas un concepto o unidad de interpretación.

A pesar de ello, este último autor (Barceló 1988: 51) advierte que la Estadística no añade ni un ápice a la Verdad a las construcciones interpretativas, sino que constituye una manera de obtener información que de otro modo permanecería oculta.

Esta relatividad hace recomendable la aplicación de distintos procedimientos en el análisis de unos mismos datos. Así lo cree A.J. Lorrio (1991: 112) que restringiéndose al ámbito de la clasificación arqueológica, hace hincapié en el interés de utilizar diferentes técnicas estadísticas para así poder realizar una aproximación, lo más objetiva posible, en un campo donde lo subjetivo constituye usualmente la norma general.

Precisamente los desarrollos más espectaculares en lo relativo a la metodología estadística han tenido lugar en el campo de la Taxonomía y la Clasificación. Esto se debe a que una de las mayores preocupaciones del arqueólogo se centra en la tipología, con la definición objetiva y científica que posteriormente se puedan comparar y contrastar (Contreras F. y col. 1991: 65).

En el caso que nos ocupa una primera observación de la Tableta de Cerro Macareno recuerda a una matriz de datos binarios. A partir de aquí las técnicas multivariantes susceptibles de aplicación son variadas.

Para comenzar la exploración, es necesario preguntarse si las muescas grabadas están colocadas aleatoriamente. En este caso no sería posible predecir su valor con ninguna técnica. En la realización de esta tarea nuestras fuentes de información son muy limitadas, tanto que deben restringirse únicamente a las que se desprendan de la propia Tableta.

Así las cosas, es posible plantearse que cada una de las casillas de la Tableta debe ser independiente del resto, si sus valores se hubieran puesto al azar. Teóricamente no sería posible predecir el valor de cada casilla en función de las del resto de la Tableta.

Dicho de otra forma: Si no conociéramos el valor de una casilla ¿con qué éxito puede adivinarse su valor, a partir de la información del resto de la Tableta? ¿Y qué ocurriría si se repitiese esta operación con todas y cada una de las casillas de la Tableta?

Para dar respuesta a estas preguntas y realizar una exploración de la información contenida en la Tableta se han utilizado varios procedimientos multivariantes. Esta aproximación es corriente ya que son numerosos los investigadores que utilizan el análisis multivariante para el tratamiento de los datos recogidos de material cerámico.

Por ejemplo, C. Risquez C. y col. (1991: 83-98) aplican un análisis de conglomerados con el objeto de lograr una clasificación de la cerámica hallada en el Cerro de la Plaza de Armas de Puente (Jaén) durante los años 1985 y 1986. El análisis se basó en variables obtenidas de fragmentos de bordes en forma de distancias. Más tarde utilizaron un análisis discriminante con el fin de confirmar la clasificación obtenida. 
A.J. Lorrio (1991: 99-112) aplica diferentes técnicas multivariantes a un grupo de 36 vasijas de cerámica gris procedente de la necrópolis de Medellín (Badajoz). El autor utiliza varios procedimientos de análisis de conglomerados con el objeto de validar una clasificación previa definida de forma intuitiva.

Fernández V. y col. (1991: 123-131) realizan un análisis sobre datos de cerámicas y cuencos de bronce de la necrópolis meroítica antigua de Amir Abdallah a $200 \mathrm{kms}$. de la frontera con Egipto. Los autores analizan las correlaciones de los datos de estos objetos con los obtenidos de aspectos básicamente cualitativos de la necrópolis en la que fueron hallados. Para ello utilizan métodos multivariantes como el análisis de correspondencias.

Cereijo M. A. y col.(1991: 143-151) utilizan un análisis de correspondencias con el objeto de determinar los factores que podrían afectar a la posición cuantitativa de arqueofaunas en 15 yacimientos medievales. Estos autores utilizan más tarde un análisis discriminante para averiguar si resulta más significativa la división de la muestra por grupos culturales o por una agrupación geográfica.

Una diferencia sustancial de estos estudios con respecto al presente, reside en que en este último nos centramos únicamente en una sola pieza cerámica. En esta pieza reside toda la información objeto de análisis.

\section{MÉTODO}

Con el propósito de comprobar la hipótesis de aleatoriedad de la información contenida en la Tableta, previamente se realizó la codificación de la Tableta siguiendo los siguientes criterios:

1. Se asignó valor " 1 " a las casillas que contenían una raya vertical, y un " 2 " a aquéllas que contenían una horizontal.

2. Como había casillas con un nivel de dificultad apreciable para asignarles uno de estos valores, se incluyeron dos nuevas posibilidades: Un "3" para la oblicua izquierda ( $\backslash$ ) y un "4" para la oblicua derecha (/).

3. Aquellas casillas que no tenían representada ninguna muesca han sido codificadas como " 5 ".

Dos jueces realizaron una codificación independiente. Éstos recibieron instrucciones de utilizar los códigos 3 ó 4 únicamente en el caso de que fueran incapaces de asignarles algún otro. Consecuencia de ello es que ninguno de los dos jueces tuvo que recurrir a codificar alguna de las casillas como oblicua izquierda.
Una vez codificada la Tableta, a cada casilla le fueron asignadas 26 variables, que corresponden a los valores que tienen sus 13 casillas contiguas por la derecha y a sus 13 por la izquierda.

Como ejemplo, tomamos la casilla de la segunda fila y tercera columna (Fig. 1). A esta casilla es posible asignarle dos variables relacionadas con las casillas que tiene por la izquierda (Izq1 y Izq2) y dos variables relacionadas con las casillas que tiene por la derecha (Der1 y Der2).

En el caso del ejemplo, la variable Izq1 contiene la información del valor de la casilla contigua por la izquierda. Para la casilla de la fila 2 y tercera columna, la casilla contigua por la izquierda toma valor 1 , por ser una casilla vertical.

Para la misma casilla del ejemplo la variable Izq2 toma valor 2. Es decir la segunda contigua por la izquierda, es una casilla horizontal.

Para las variables Der1 y Der2, el procedimiento a seguir es el mismo. Para la casilla de la fila 2 y tercera columna la contigua a su derecha es una casilla horizontal. Esto equivale a decir que la variable Der1 toma valor 2. De la misma forma, tenemos que la variable Der2 tiene valor 1, al ser la segunda contigua por la derecha una casilla con muesca vertical.

Esta forma de proceder ha permitido asignar a cada casilla de la Tableta 26 variables con las que poder realizar este estudio. Existen otras variaciones similares a la utilizada, tales como tomar las casillas de arriba y abajo, las oblicuas, u otras posibilidades más complejas. Estas posibilidades no van a ser abordadas en este estudio.

A pesar de lo expuesto existe una obviedad que no puede ser ignorada: ninguna casilla tiene completa la información de las 26 variables. Existe una limitación estructural dada por la propia forma de la Tableta.

En el ejemplo comentado anteriormente, no existe ningún valor para la variable Izq3: su posición queda fuera de la Tableta. En otras palabras, no existe una casilla contigua en 3 posiciones a la izquierda para la casilla de la segunda fila y tercera columna.

Esta dificultad ha sido tratada tomando como valores perdidos los valores de las variables siempre que se producía esta casuística. Tras ello, se han aplicado a estas variables varios procedimientos estadísticos.

Un primer acercamiento fue realizado con un análisis de correspondencias. Sobre las dimensiones obtenidas se realizó un análisis discriminante utilizando como variable dependiente a las casillas originales. Además se ha rotado la solución obtenida para simplificar la interpretación (rotación Varimax).

Por último, las funciones discriminantes fueron contrastadas con coeficientes de correlación theta y con 
comparaciones múltiples de medias por el procedimiento de Scheffé. El nivel de confianza utilizado ha sido siempre de un $95 \%$.

\section{RESULTADOS}

De las 112 casillas de la Tableta codificadas por los jueces, sólo en dos casos hubo disparidad de criterios. De esta forma, podríamos cifrar en un 98,2\% la fiabilidad interjueces.

Se representa en el gráfico (fig. 2) las dos primeras dimensiones obtenidas en el análisis de correspondencias. A simple vista aparece una marcada división en dos zonas. La zona de la izquierda en el eje de abscisas, corresponde a las dos últimas filas de la Tableta en las que aparecen un buen número de casillas en blanco.

En el mismo gráfico se observa la cercanía existente entre cuatro de las cinco casillas oblicuas, agrupadas por debajo del valor -1 del eje de ordenadas. También se aprecia en el mismo eje una mayor concentración de casillas verticales por encima del valor 0 $y$, por debajo de él, una mayor concentración de casillas horizontales.

Las dos dimensiones del gráfico, junto con el resto de las dimensiones obtenidas en el análisis de correspondencias, se han utilizado para comprobar con qué éxito podría realizarse una reclasificación de las casillas. Para ello se ha aplicado sobre ellas un análisis discriminante para tratar de predecir los cuatro tipos posibles de casillas.

Tres funciones discriminantes se han obtenido con este análisis. La menos significativa estadísticamente fue la tercera $(p=0,0514)$. Estas funciones presentaban correlaciones canónicas importantes con respecto a las

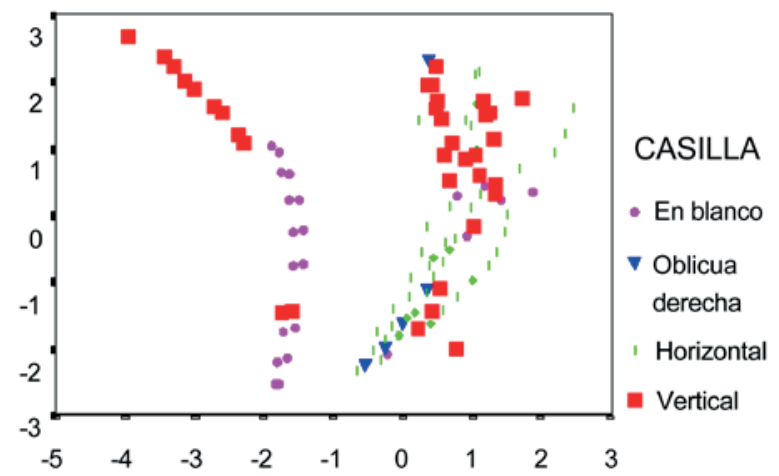

Fig. 2. Dos primeras dimensiones del análisis de correspondencias

casillas que tratan de ser clasificadas. Sus correlaciones oscilaban entre 0,70 para la primera y 0,56 para la tercera función.

La primera función establece diferencias significativas entre las casillas en blanco y el resto de las casillas. Las diferencias generadas por la segunda función se establecen entre las casillas oblicuas y el resto de las casillas. Otro tanto ocurre con la tercera función discriminante, pero esta vez las diferencias se establecen con respecto a las casillas horizontales.

Teniendo en cuenta que los cuatro valores que contiene la Tableta tenían igual probabilidad de ser elegidos, el análisis realizado ha conseguido clasificar correctamente el 76,8\% de las casillas de la Tableta.

Tal y como se observa en la Tabla de Clasificación (Figura 3), las casillas horizontales, en blanco y las oblicuas han sido clasificadas con un éxito similar que ronda el $80 \%$ de aciertos. Las casillas verticales fueron clasificadas correctamente en un $71,4 \%$, mostrando

\begin{tabular}{|c|c|c|c|c|c|c|c|}
\hline & & \multicolumn{5}{|c|}{ Predicción } \\
\hline & & Casilla & Vertical & Horizontal & Oblicua & Blanco & Total \\
\hline \multirow{8}{*}{ 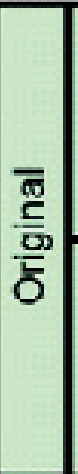 } & \multirow{4}{*}{ 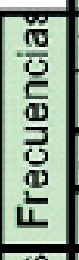 } & Vertical & 25 & 6 & 1 & 3 & 35 \\
\hline & & Horizontal & 7 & 38 & 2 & 1 & 48 \\
\hline & & Oblicua & 0 & 1 & 4 & 0 & 5 \\
\hline & & Blanco & 0 & 5 & 0 & 19 & 24 \\
\hline & \multirow{4}{*}{ 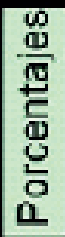 } & Vertical & 71.4 & 17.1 & 2.9 & 8.6 & 100.0 \\
\hline & & Horizontal & 14.6 & 79.2 & 4.2 & 2.1 & 100.0 \\
\hline & & Oblicua & 0.0 & 20.0 & 80.0 & 0.0 & 100.0 \\
\hline & & Blanco & 0.0 & 20.8 & 0.0 & 79.2 & 100.0 \\
\hline
\end{tabular}

Fig. 3. Tabla de clasificación de valores originales/predichos 
Fig. 4. Tabla de errores en la predicción VALOR ORIGINAL/VALOR PREDICHO

\begin{tabular}{|c|c|c|c|c|c|c|c|c|c|c|c|c|c|c|}
\hline & 1 & 2 & 3 & 4 & 5 & 6 & 7 & 8 & 9 & 10 & 11 & 12 & 13 & 14 \\
\hline 1 & & & $2 / 1$ & & $5 / 2$ & & & & & & & & & \\
\hline 2 & $2 / 1$ & & & & & & & $2 / 4$ & & & $1 / 2$ & & & \\
\hline 3 & & & $1 / 4$ & & & & & & & & & & & \\
\hline 4 & & $2 / 1$ & $2 / 1$ & & $1 / 2$ & & & $5 / 2$ & & & $1 / 2$ & $1 / 2$ & & \\
\hline 5 & $2 / 4$ & & & $2 / 1$ & & & $5 / 2$ & $1 / 2$ & & & & & & \\
\hline 6 & $1 / 2$ & & & $2 / 1$ & & $1 / 5$ & $5 / 2$ & & $2 / 5$ & & & & $5 / 2$ & $4 / 2$ \\
\hline 7 & $2 / 1$ & & & & & & & & & & $1 / 5$ & & & \\
\hline 8 & & & & & & & & & & & $1 / 5$ & & & \\
\hline & & $\begin{array}{l}\text { cal } \\
\text { izonta }\end{array}$ & & & & & & $\begin{array}{l}4= \\
5=\end{array}$ & $\begin{array}{l}\text { icua } \\
\text { illa e }\end{array}$ & $\begin{array}{l}\text { echa } \\
\text { lanco }\end{array}$ & & & & \\
\hline
\end{tabular}

una tendencia a ser confundidas preferentemente con las horizontales. Esto ocurrió en seis casos.

En siete casos se asignaron erróneamente casillas horizontales al hacerlo como verticales. Y los cinco únicos errores cometidos al clasificar las casillas en blanco fueron horizontales.

El hecho de que las casillas verticales sean menos predecibles las convierte en las más significativas con respecto al suceso registrado por el alfarero. Este hecho, unido a que las casillas en blanco sólo han sido confundidas con las casillas horizontales, nos obliga a plantear la hipótesis de que las casillas horizontales y las blancas son equivalentes.

Esto nos explicaría la existencia del grupo de casillas en blanco de las dos últimas filas. Esta zona de casillas sólo está interrumpida precisamente por dos casillas verticales de la novena columna. Así, podríamos considerar que el alfarero sólo ha registrado en las dos filas la información más significativa; esto es, la referida a las casillas verticales, y ha omitido por descuido o desidia las casillas horizontales.

A pesar de estos errores, podemos calificar la validez de la clasificación de las casillas, a partir de sus contiguas, como alta. En este sentido obtenemos una relación entre los valores de las casillas y los valores predichos de 0,76 (coeficiente de contingencia).

De esta forma queda demostrado que las casillas no se distribuyen al azar, al estar relacionadas unas con otras de una manera suficientemente intensa como para predecir sus valores a partir de estas relaciones. ¿Pero qué ocurre con aquellas casillas que no fueron bien clasificadas?
Estas nos dan información de aquello que no se puede predecir por la inercia de la propia Tableta (Véase la tabla de errores de predicción de la figura 4). En este caso se trata de una información muy relevante: aquella información que aporta la realidad exterior y que ha tratado de ser captada con la Tableta. Si el suceso que se recoge en la Tableta hubiera sido simple o predecible, ésta hubiera sido innecesaria.

Comencemos por los tres aspectos más fácilmente predecibles $\mathrm{y}$, por tanto, más evidentes:

1. En la parte derecha de la Tableta lo más esperado son casillas horizontales en las 6 primeras filas, y blancas en las 2 últimas. Sirva como ejemplo la décima columna. Ésta es la única en la que no se ha cometido ningún error de predicción. En ella, además de la casilla oblicua, encontramos casillas horizontales, salvo las dos casillas en blanco correspondientes a las dos últimas filas.

2. Las filas séptima y octava tienen una distribución de valores que las hace diferentes de las del resto de la Tableta. Para ellas lo más esperado son casillas verticales hasta la quinta columna y el resto casillas en blanco. La fila octava ilustra esta observación al contener sólo un error en la predicción.

3. Puede observarse en las 6 primeras filas un predominio por la izquierda de casillas verticales y un predominio por la derecha de las casillas horizontales. Un claro ejemplo de ello lo constituye la fila tercera. Ésta contiene únicamente un error en la predicción y destaca por su 
distribución de casillas básicamente verticales hasta la sexta columna. A partir de ahí el predominio recae en las casillas horizontales.

Como era de esperar, en la zona izquierda de la Tableta se observan errores debidos al efecto de la irrupción de casillas horizontales en una zona con predominio de verticales. En este sentido, cinco de los nueve errores son por asignación de casillas verticales a casillas horizontales.

En la zona central de la Tableta de errores hay seis casillas, próximas entre sí, cuyos errores parecen haberse producido por efecto de las casillas blancas que hay a la altura de la séptima columna en las filas quinta y sexta. La mitad de estos errores son cometidos al asignar casillas horizontales a casillas en blanco. Por el contrario, dos errores son debidos al asignar casillas en blanco erróneamente.

Por la derecha, los errores se producen por la intrusión de casillas verticales en una zona de blancas y, en otro caso, por la aparición de casillas verticales en una zona de horizontales.

El último error que vamos a comentar es el producido en la intersección de la primera fila y de la quinta columna. Este error resalta las marcadas monotonías de los valores de esta columna y de esta fila, la primera saturada de casillas verticales y la segunda saturada de valores horizontales.

\section{CONCLUSIONES}

Las muescas contenidas en la Tableta de Cerro Macareno permiten la aplicación de un sistema de codificación de datos. Este proceso puede hacerse, además, con una fiabilidad elevada. Los resultados de la codificación permiten la aplicación de posteriores análisis estadísticos sobre la información recogida.

Con los análisis estadísticos aplicados se ha conseguido rechazar la hipótesis de que las muescas de la Tableta se distribuyan al azar. Ha sido posible predecir el valor de cada casilla con un acierto tres veces superior al que se hubiera obtenido clasificándolas al azar.

Por otra parte, tanto la observación directa como la exploración realizada, denotan la posibilidad de que sus dos últimas filas hayan sido un añadido realizado en un segundo momento durante la creación de la Tableta. Quizá, el creador de la pieza, una vez preparado el bloque de barro y marcadas las filas, tratara de aprovechar el espacio sobrante.

También podría ser que se ha obviado completar esta zona por algún motivo desconocido, o que se trate de un intento de realizar un arreglo para llegar a un diseño preconcebido. Si esto es así, podría tratarse de una pieza defectuosa que no se ha completado en sus últimas casillas, dejando el autor casillas en blanco.

Sin embargo, hay que recordar el hecho de que las casillas en blanco únicamente han sido confundidas con horizontales al hacer la reclasificación. Esto nos permite formular la hipótesis de que las casillas horizontales y las blancas representan un único fenómeno, es decir, la ausencia del suceso que trataba de ser registrado con la Tableta.

Esta hipótesis es coherente con la idea de que el autor ha obviado señalar esta zona con muescas horizontales, por no aportar éstas nada nuevo a la Tableta. Este argumento se ve reforzado con la irrupción poco previsible de dos casillas verticales en la zona de blancas a la altura de la onceava columna.

En otras palabras, las casillas verticales pueden estar señalando la presencia del fenómeno, y las casillas horizontales y blancas mostrando la ausencia del fenómeno.

Por otra parte, se percibe una tendencia a una mayor concentración de casillas verticales en la parte izquierda de la Tableta, y en la zona derecha un claro predominio de casillas horizontales. De acuerdo con los resultados obtenidos, a partir de la sexta columna podemos establecer una división imaginaria de la Tableta.

Sin embargo la peculiaridad de la quinta columna, en la que todas sus casillas, salvo la primera, son verticales, se presta mejor para cubrir la función divisoria de la Tableta. Esta división tan clara de la Tableta nos hace pensar en la posibilidad de que pudiera estar representando algún fenómeno de tipo espacial, representando la fila 1 y la columna 5 dos ejes de coordenadas.

Como síntesis decir que hay dos hipótesis generales que podrían dar sentido al contenido de la Tableta. La primera, que muestra evidencias observables, que se trate de algún registro de información espacial. La segunda, que requeriría una aproximación a su estudio distinta, que se trate de algún apunte de la distribución de algún tipo de suceso en el tiempo.

En cualquier caso, dada la simplicidad de la información recogida por la Tableta no nos inclinamos a pensar que pueda tratarse de algún tipo de escritura.

\section{BIBLIOGRAFÍA}

BARCELÓ, J.A. (1988): “Introducción al razonamiento estadístico aplicado a la arqueología: un análisis de las estelas antropomorfas de la península ibérica", Trabajos de Prehistoria 45: 51-85. 
- (1991): "El uso de sistemas expertos en la interpretación de clasificaciones estadísticas multidimensionales", Complutum 1: 41-51.

CEREIJO, M. A.; HERRANZ, M.A. y PATON, D. (1991): "Caracterización de arqueofaunas medievales a partir de análisis multivariante", Complutum 1: $143-151$.

CARRIAZO, J. DE M. (1973): Tartesos y el Carambolo. Univ. y Acad. Madrid.

CONTRERAS, F.; MOLINA, F. y ESQUIVEL, J.A. (1991): "Propuesta de una metodología para el estudio tipológico de los complejos arquelógicos mediante análisis multivariante", Complutum 1: 65-82.

FERNANDEZ, F.; CHASCO, V. y OLIVA, D. (1979): "Excavaciones en el "Cerro Macareno", La Rinconada (Sevilla)”, Noticiario Arqueológico Hispánico 7: 10-93.
FERNANDEZ, V. y GARCIA, M. (1991): “Tratamiento informático de datos funerarios cualitativos: análisis de correspondencias y algoritmo ID3 de Quinlan”, Complutum 1: 123-131.

FERNANDEZ-CHICARRO, C. (1974): “Adquisiciones del Museo Arqueológico de Sevilla”, Revista Bellas Artes 74, no 33: 31.

IFRAH, G. (1998): Historia Universal de las cifras. Ed. Espasa Calpe. Madrid. $3^{\text {a }}$ Edición.

LORRIO, A.J. (1991): "Clasificación automática de formas cerámicas completas: un estudio comparativo de diversos métodos multivariantes", Complutum 1: 99-112.

POSAC, C. (1975): "Los Algarbes (Tarifa). Una necrópolis de la Edad del Bronce", Noticiario Arquelógico Hispánico 4: 85-119.

RISQUEZ, C.; HORNOS, F.; RUIZ, A. y MOLINOS, M. (1991): "Análisis multivariante: una propuesta de tipología contextualizada”, Complutum 1: 83-98. 